\title{
ABSCESO PERIRRENAL: FACTORES ASOCIADOS A SU DESARROLLO Y MORTALIDAD
}

\author{
Efraín Maldonado-Alcaraz, Guillermo Ixquiac-Pineda, Virgilio López-Sámano y Eduardo Serrano- \\ Brambila.
}

Servicio de Urología. Hospital de Especialidades Centro Médico Nacional Siglo XXI. México. Distrito Federal. México.

Resumen.- OBJETIVO: Determinar los factores predisponentes para la formación de abscesos perirrenales y encontrar eventos asociados a una evolución desfavorable.

MÉTODOS: Realizamos un estudio clínico, descriptivo, retrospectivo y transversal que incluyó 23 pacientes con diagnóstico de absceso perirrenal ingresados en nuestro hospital.

RESULTADOS: Las entidades clínicas asociadas con el absceso perirrenal fueron: Diabetes mellitus en $65.2 \%$, antecedente de litiasis renal en $43.47 \%$ y el antecedente de cirugía urológica en $17.38 \%$. Al ingreso, la hemoglobina mayor a $10.5 \mathrm{~g} / \mathrm{dl}$ y leucocitos menores a $15 \times 10^{3} / \mu \mathrm{L}$ se asociaron con mayor frecuencia a nefrectomía y las plaquetas menores a $140 \times 10^{3}$ / $\mu \mathrm{L}$ se asociaron a choque séptico. La mortalidad general fue de $8.69 \%$ y $78.3 \%$ requirieron nefrectomía. Los pacientes que murieron presentaron al ingreso fiebre,
Efraín Maldonado-Alcaraz

Av. Cuauhtémoc 330

Col. Doctores

C.P. 06760. (México D. F.). urologia_maldonado@yahoo.com.mx

Trabajo recibido: 10 de enero 2007 anemia, leucocitosis mayor a $16 \times 10^{3} / \mu \mathrm{L}$, plaquetas menores a $130 \times 10^{3} / \mu \mathrm{L}$, sodio sérico menor a 125 $\mathrm{mEq} / \mathrm{L}$, requirieron nefrectomía y presentaron choque séptico.

CONCLUSIONES: En nuestra serie un buen porcentaje de pacientes con absceso perirrenal son portadores de diabetes mellitus y/o litiasis renal. El nivel de hemoglobina y de leucocitos se asoció con la pérdida de la unidad renal, la trombocitopenia se asoció a choque séptico y la hiponatremia con la mortalidad.

Palabras clave: Absceso perirrenal. Absceso retroperitoneal. Choque séptico.

Summary.- OBJECTIVES: To determine predisposing factors in perinephric abscesses and to find events associated with unfavourable outcome.

METHODS: We carried out a clinical, descriptive, retrospective and cross-sectional study, including 23 patients diagnosed of perinephric abscess admitted to our hospital.

RESULTS: In patients with perinephric abscess, cli-nical charts included diabetes mellitus in $65.2 \%$, history of nephrolithiasis in $43.47 \%$ and history of urological surgery in $77.38 \%$.

On hospital admission, haemoglobin greater than 10.5 $\mathrm{g} / \mathrm{dL}$ and white blood cell count lower than $15 \times 10^{3} /$ $\mu \mathrm{L}$ were associated with nephrectomy, and platelet count lower than $140 \times 10^{3} / \mu \mathrm{L}$ with septic shock. General mortality was $8.69 \%$, and $78.3 \%$ if patient required nephrectomy. Patients who died had fever, anaemia, white blood cell count greater than $16 \times 10^{3} / \mu \mathrm{L}$, platelet count lower than $130 \times 10^{3} / \mu \mathrm{L}$, and hyponatremia of $125 \mathrm{mEq} / \mathrm{L}$ or lower at hospital admittance, and all of them had septic shock and required nephrectomy. 
CONCLUSIONS: In our series, a higher percentage of patients had diabetes mellitus and/or nephrolithiasis. Haemoglobin level and white blood cells count were associated with loss of the renal unit, thrombocytopenia was associated with septic shock and hyponatremia with mortality.

Keywords: Perinephric abscess. Retroperitoneal abscess. Septic shock.

\section{INTRODUCCIÓN}

Las infecciones supurativas retroperitoneales que involucran el riñón son difíciles de diagnosticar y de tratar. Tradicionalmente los abscesos perirrenales han sido asociados con mortalidad y morbilidad significativas. A pesar de un drenaje quirúrgico agresivo la mortalidad puede variar del $39-56 \%$ (1). Algunos factores que predisponen al desarrollo de abscesos perirrenales son: Litiasis renal, infección urinaria, reflujo vesico-ureteral, trauma, diabetes mellitus, neoplasias y enfermedad hepática. El $40 \%$ tienen múltiples factores de riesgo asociados. En pacientes sin anormalidades de las vías urinarias el factor de riesgo más importante es la diabetes mellitus y en $39 \%$ son abscesos intrarrenales, $19 \%$ perirrenales e intrarrenales y $42 \%$ únicamente perirrenales (5).

La infección urinaria ascendente, la diseminación hematógena, o la contaminación por una infección adyacente, son los mecanismos más comunes en la génesis de un absceso perirrenal (6). La duración de los síntomas antes del diagnóstico es en promedio de 11 días y solamente el 35\% son diagnosticados correctamente al ingreso hospitalario debido a la falta de datos clínicos específicos (7). La presentación clínica es principalmente con fiebre en el $79 \%$, otros síntomas incluyen dolor abdominal y en el flanco, síntomas urinarios y malestar general (8). La estancia hospitalaria de estos pacientes es en promedio 11 días (1-4).

No existen exámenes de laboratorio específicos para el diagnóstico de absceso perirrenal, pero con frecuencia existe anemia, leucocitosis, leucocituria y bacteriuria (9). Siegel y cols. presentan una serie de 52 pacientes con abscesos renales en un periodo de 9 años, encontrando leucocitosis (en promedio 15 $\mathrm{X} 10^{3} / \mu \mathrm{L}$ ), elevación de creatinina (en promedio 1.7 $\mathrm{mg} / \mathrm{dL}$ ) y piuria en el examen general de orina en el $92 \%$ de los pacientes. Los cultivos fueron positivos en el $71 \%$ de orina, $28 \%$ de sangre y $77 \%$ de muestras de aspirado (8).

Los abscesos perirrenales pueden ser causados por diferentes tipos de microorganismos, de- sarrollándose cepas de gram negativos aerobios en $52 \%$ de los casos, predominando Escherichia coli. El Staphylococcus aureus se encuentra en $29 \%$ y los anaerobios en $17 \%$. Existen otros microorganismos menos frecuentes, como Salmonella enteritidis (10). y Candida albicans (11). En $71 \%$ de los casos se desarrolla sólo un microorganismo y en $29 \%$ dos o más (12-13).

Actualmente el ultrasonido (USG) y la tomografía computada (TC) son estudios disponibles en la mayoría de los hospitales, permitiendo $82-95 \%$ de sensibilidad en el diagnóstico. El USG es el método más rápido y barato, pero la TC actualmente es el procedimiento de elección como parte de la complementación diagnóstica $(8,12,14,15)$.

Los abscesos perirrenales se deben manejar inicialmente con antibióticos parenterales. La selección de un tratamiento empírico inicial depende de la sospecha del origen de la infección, si esta es hematógena el Staphylococcus penicilina resistente es el microorganismo patógeno más frecuente y debe tratarse con penicilinas isoxazólicas como la dicloxacilina, en los casos en que existiera hipersensibilidad a la penicilina se recomienda el uso de cefalosporinas o vancomicina. En los casos de infección en forma ascendente o por alguna anormalidad del tracto urinario, el tratamiento empírico de elección es con cefalosporinas de tercera generación o aminoglucósidos $(8,11,16)$.

El drenaje de los abscesos ya sea de manera percutánea o a través de una cirugía abierta es la modalidad terapéutica más ampliamente aceptada. A pesar de que se ha sugerido el drenaje percutáneo para tratar los abscesos en pacientes inmunocomprometidos, los que no responden al tratamiento antimicrobiano y los menores a $5 \mathrm{~cm}$. de diámetro, el drenaje quirúrgico abierto sigue siendo la mejor opción para los abscesos mayores de $5 \mathrm{~cm}$ de diámetro. Algunos pacientes seleccionados con abscesos menores de tres centímetros de bajo riesgo pueden ser tratados únicamente con antibióticos parenterales, con seguimiento estrecho por USG $(1,15,17,18)$.

Bickel y cols. (19) reportó un caso de absceso perirrenal que fue tratado por laparoscopia. Menciona en este reporte que a pesar de no ser el mejor abordaje, es una opción cuando el diagnóstico se realiza durante la exploración laparoscópica, pudiéndose tratar en forma satisfactoria por esta vía.

Hasta una tercera parte de los pacientes pueden requerir nefrectomía parcial o total durante su estancia hospitalaria, como parte del abordaje terapéutico inicial o de manera tardía $(1,20)$. 
Los principales objetivos de este trabajo son describir la evolución y establecer los factores predisponentes para la formación de abscesos perirrenales, determinar la mortalidad relacionada con el absceso perirrenal, y encontrar eventos asociados a una evolución desfavorable en los pacientes ingresados en nuestro hospital.

\section{MATERIAL Y MÉTODOS}

Se realizó un estudio clínico, retrospectivo, transversal, descriptivo y observacional de 23 expedientes de pacientes adultos de ambos sexos con diagnóstico de absceso renal, perirrenal o retroperitoneal tratados en el servicio de urología del Hospital de Especialidades Centro Médico Nacional Siglo XXI que se atendieron durante el período comprendido entre Enero del 2001 y Enero del 2006. Excluyendo del estudio a los pacientes con expediente depurado o no localizable. Se recabaron los datos referentes a antecedentes, valores de laboratorio a su ingreso y evolución hospitalaria, con el objetivo de encontrar factores asociados a evolución desfavorable. El proyecto fue aprobado por el comité local de investigación. Los datos se tabularon en formato Excel ${ }^{\circledR}$, se analizaron los resultados con el paquete estadístico SPSS 11.0.

\section{RESULTADOS}

Se revisaron los expedientes de 34 pacientes con diagnóstico de absceso perirrenal que ingresaron a nuestro hospital en el periodo comprendido de enero de 2001 a enero de 2006, de los cuales solo 23 reunieron toda la información requerida para la inclusión al estudio, el resto de pacientes se encontró que habían sido dados de alta vivos, siendo depurados los expedientes. Del total, 16 pacientes $(70 \%)$ fueron mujeres y 7 pacientes $(30 \%)$ hombres, con un promedio de edad de 53 años (22-75 años).

El índice de masa corporal promedio en estos pacientes fue de $27.83 \mathrm{~kg} / \mathrm{m}^{2}$, presentándose algún grado de sobrepeso en el $60.8 \%$ de los pacientes. La diabetes mellitus se presentó en 15 pacientes $(65.2 \%)$, el antecedente de litiasis renal se presentó en 10 pacientes $(43.47 \%)$, en 2 pacientes $(8.69 \%)$ se encontraron alteraciones anatómicas de las vías urinarias (ectopia renal cruzada y estenosis de la unión uretero-piélica secundaria a litiasis, respectivamente). Cuatro pacientes (17.38\%) tenían el antecedente de cirugía urológica en los tres meses previos a su ingreso. Un paciente tenía antecedente de tuberculosis renal. La fiebre al ingreso se presentó en 13 pacientes $(56.52 \%)$.
En cuanto a los laboratorios a su ingreso, la Hemoglobina $(\mathrm{Hb})$ media fue de $10.35 \mathrm{~g} / \mathrm{dL}$ y la cuenta de leucocitos media fue de $17 \times 10^{3} / \mu \mathrm{L}$, se presentó trombocitopenia en 10 pacientes $(43.47 \%)$, la glucemia tuvo una media de $256 \mathrm{mg} / \mathrm{dL}$, el sodio sérico promedio fue de $134 \mathrm{mEq} / \mathrm{L}$. El sodio sérico menor a $130 \mathrm{mEq} / \mathrm{L}$ se presentó en 10 pacientes y se asoció en $90 \%$ a nefrectomía y al requerimiento de terapia intensiva en $60 \%$. La creatinina se presentó con una media de $2.26 \mathrm{mg} / \mathrm{dL}$. Doce pacientes $(52.17 \%)$ tuvieron tiempos de coagulación prolongados.

Al realizar el análisis estadístico, la hemoglobina mayor a $10.5 \mathrm{~g} / \mathrm{dL}$ y la cuenta leucocitaria menor a $15 \times 10^{3} / \mu \mathrm{L}$ fueron factores asociados a la nefrectomía ( $p=0.042$ y $p=0.01$ respectivamente). Ninguno de los factores estudiados se asoció significativamente al requerimiento de estancia en terapia intensiva o al desarrollo de neumonía nosocomial. La cuenta plaquetaria menor a $140 \times 10^{3} / \mu \mathrm{L}$ se asoció a choque séptico de manera significativa $(p=$ $0.027)$. De los valores de laboratorio asociados con mortalidad únicamente el sodio fue estadísticamente significativo (Tabla I).

Se realizó nefrectomía en 18 pacientes (78.3\%). El drenaje promedio en 24 horas fue de $71 \mathrm{~mL}$, y se retiró en promedio a los 8 días.

Los cultivos del material purulento desarroIlaron predominantemente Escherichia coli (43.47\%) (Tabla II). Ocho pacientes desarrollaron choque séptico $(34.8 \%)$, Diez pacientes $(43.47 \%)$ requirieron terapia intensiva, con una estancia promedio en terapia de 12 días. La neumonía nosocomial se presentó en 8 pacientes $(34.78 \%)$.

La estancia promedio de los pacientes fue de 20 días, con un mínimo de 5 y un máximo de 47 días.

Dos pacientes fallecieron $(8.69 \%)$, los dos diabéticos con más de 10 años de evolución, uno de ellos con antecedente de litiasis renal, ambos de presentaron a su ingreso fiebre mayor a $38.5^{\circ} \mathrm{C}$, anemia, leucocitosis mayor a $16 \times 10^{3} / \mu \mathrm{L}$, trombocitopenia menor a $130 \times 10^{3} / \mu \mathrm{L}$ y sodio sérico menor a $125 \mathrm{mEq} / \mathrm{L}$; ambos pacientes requirieron nefrectomía y presentaron neumonía nosocomial asociada a choque séptico, uno con 26 días de estancia en terapia intensiva y otro con 8 días.

\section{DISCUSIÓN}

Los abscesos perirrenales se asocian con morbilidad y mortalidad elevadas, debido a que históricamente ha sido difícil el diagnóstico y el tratamiento 
(1), los aspectos que caracterizan esta entidad son el diagnóstico tardío y la necesidad de un tratamiento temprano. En la actualidad con mejores estudios de imagen es más preciso el diagnóstico de patologías renales y retroperitoneales. Los abscesos perirrenales se presentan a una edad promedio de 46 años, con un predominio de mujeres del $80 \%$ según Shu y cols. (5), en algunas series el predominio fue del género masculino en $52 \%$ Meng y cols. (4). En nuestra serie se encontró una edad promedio de 53 años predominando las mujeres en el $70 \%$.

Se han identificado factores que predisponen al desarrollo de abscesos perirrenales, siendo los mas relevantes: diabetes mellitus, litiasis renal, presencia de infección urinaria, reflujo vesico-ureteral, trauma, neoplasias y enfermedad hepática (1-4). El 40\% de los pacientes tienen múltiples factores de riesgo aso- ciados (1-4). En nuestro estudio se encontró diabetes mellitus en 15 pacientes (65.2\%). Los pacientes con más de 10 años de diagnóstico de la diabetes requirieron con mayor frecuencia de nefrectomía 188 vs $71 \%$ ), manejo en terapia intensiva (75 vs $43 \%$ ) y neumonía nosocomial asociada a choque séptico (63 vs $29 \%$ ) aunque ninguna de estas diferencias fue estadísticamente significativa, creemos que debido a que la muestra es pequeña.

La duración de los síntomas previo al diagnóstico es en promedio de 11 días y solo el 35\% de los pacientes son diagnosticados correctamente al ingreso hospitalario debido a la falta de datos clínicos específicos (7). Con el uso rutinario del USG y la TC en servicios de urgencias se han mejorado estas cifras (1). La presentación clínica de los pacientes es principalmente con fiebre en el $79 \%$ de los pacientes,

TABLA I. CORRELACIÓN DE LOS DATOS DE LABORATORIO ASOCIADOS A EVENTOS DESFAVORABLES EN LOS PACIENTES CON DIAGNÓSTICO DE ABSCESO RETROPERITONEAL.

\begin{tabular}{|c|c|c|c|c|c|}
\hline & & $\begin{array}{c}\text { Hemoglobina al } \\
\text { ingreso }\end{array}$ & $\begin{array}{c}\text { Leucocitos al } \\
\text { ingreso }\end{array}$ & $\begin{array}{c}\text { Plaquetas al } \\
\text { ingreso }\end{array}$ & $\begin{array}{c}\text { Sodio sérico al } \\
\text { ingreso }\end{array}$ \\
\hline $\begin{array}{l}\text { Requirió } \\
\text { nefrectomía } \\
n=18\end{array}$ & $\begin{array}{l}\mathrm{NO} \\
\mathrm{SI}\end{array}$ & $\begin{array}{l}9.3 \mathrm{~g} / \mathrm{dL} \\
10.5 \mathrm{~g} / \mathrm{dL} \\
p=0.042\end{array}$ & $\begin{array}{c}21.9 \times 103 / \mathrm{mL} \\
15.4 \times 103 / \mathrm{mL} \\
p=0.01\end{array}$ & $\begin{array}{c}335 \times 103 / \mathrm{mL} \\
239 \times 103 / \mathrm{mL} \\
p=\text { N.S. }\end{array}$ & $\begin{array}{c}133 \mathrm{mEq} / \mathrm{L} \\
133 \mathrm{mEq} / \mathrm{L} \\
p=\text { N.S. }\end{array}$ \\
\hline $\begin{array}{l}\text { Requirió } \\
\text { Terapia Intensiva } \\
N=10\end{array}$ & $\begin{array}{l}\mathrm{NO} \\
\mathrm{SI} \\
\mathrm{p}\end{array}$ & $\begin{array}{c}10.0 \mathrm{~g} / \mathrm{dL} \\
10.75 \mathrm{~g} / \mathrm{dL} \\
p=N . S .\end{array}$ & $\begin{array}{c}18.61 \times 103 / \mathrm{mL} \\
14.57 \times 103 / \mathrm{mL} \\
p=\text { N.S. }\end{array}$ & $\begin{array}{c}321 \times 103 / \mathrm{mL} \\
180 \times 103 / \mathrm{mL} \\
p=\text { N.S. }\end{array}$ & $\begin{array}{c}134.0 \mathrm{mEq} / \mathrm{L} \\
133.5 \mathrm{mEq} / \mathrm{L} \\
p=\text { N.S. }\end{array}$ \\
\hline $\begin{array}{l}\text { Desarrolló neu- } \\
\text { monía nosoco- } \\
\text { mial }\end{array}$ & $\begin{array}{l}\mathrm{NO} \\
\mathrm{SI} \\
\mathrm{p}\end{array}$ & $\begin{array}{l}10.2 \mathrm{~g} / \mathrm{dL} \\
10.5 \mathrm{~g} / \mathrm{dL} \\
p=\text { N.S. }\end{array}$ & $\begin{array}{l}17.2 \times 103 / \mathrm{mL} \\
16.1 \times 103 / \mathrm{mL} \\
17.6 \times 103 / \mathrm{mL}\end{array}$ & $\begin{array}{c}301 \times 103 / \mathrm{mL} \\
182 \times 103 / \mathrm{mL} \\
p=\text { N.S. }\end{array}$ & $\begin{array}{c}133 \mathrm{mEq} / \mathrm{L} \\
135 \mathrm{mEq} / \mathrm{L} \\
p=\text { N.S. }\end{array}$ \\
\hline $\begin{array}{l}\text { Desarrolló } \\
\text { choque séptico }\end{array}$ & $\begin{array}{l}\mathrm{NO} \\
\mathrm{SI} \\
\mathrm{p}\end{array}$ & $\begin{array}{l}10.0 \mathrm{~g} / \mathrm{dL} \\
10.95 \mathrm{~g} / \mathrm{dL} \\
p=\text { N.S. }\end{array}$ & $\begin{array}{c}15.46 \times 103 / \mathrm{mL} \\
p=N . S . \\
17.6 \times 103 / \mathrm{mL}\end{array}$ & $\begin{array}{c}323 \times 103 / \mathrm{mL} \\
141 \times 103 / \mathrm{mL} \\
p=0.027\end{array}$ & $\begin{array}{l}134 \mathrm{mEq} / \mathrm{L} \\
133 \mathrm{mEq} / \mathrm{L} \\
p=\text { N.S. }\end{array}$ \\
\hline $\begin{array}{l}\text { Murieron } \\
n=2\end{array}$ & $\begin{array}{l}\mathrm{NO} \\
\mathrm{SI}\end{array}$ & $\begin{array}{c}10.29 \mathrm{~g} / \mathrm{dL} \\
10.75 \mathrm{~g} / \mathrm{dL} \\
\mathrm{p}=\mathrm{N} . \mathrm{S} .\end{array}$ & $\begin{array}{c}16.89 \times 103 / \mathrm{mL} \\
16.50 \times 103 / \mathrm{mL} \\
p=\text { N.S. }\end{array}$ & $\begin{array}{c}274 \times 103 / \mathrm{mL} \\
107 \times 103 / \mathrm{mL} \\
p=\text { N.S. }\end{array}$ & $\begin{array}{l}134 \mathrm{mEq} / \mathrm{L} \\
122 \mathrm{mEq} / \mathrm{L} \\
p=0.038\end{array}$ \\
\hline
\end{tabular}

$p=<0.05$ 
asociado a otros síntomas: dolor abdominal, síntomas urinarios y malestar general (8).

Paradójicamente la hemoglobina mayor a 10.5 $\mathrm{g} / \mathrm{dL}$ se asoció a la realización de nefrectomía con mayor frecuencia $(p=0.042)$, requirieron manejo en terapia intensiva el $62.5 \%$ de estos pacientes y la mitad de ellos desarrollo neumonía nosocomial, no encontramos reportes previos que evalúen la hemoglobina.

Siegel y cols. (8) reportan la serie de 52 pacientes con abscesos renales en un periodo de 9 años, encontrando leucocitosis (en promedio $15 \mathrm{X}$ $10^{3} / \mu \mathrm{L}$ ), elevación de creatinina (en promedio 1.7 $\mathrm{mg} / \mathrm{dL}$ ) y piuria en el examen general de orina en el $92 \%$ de los pacientes. En nuestro estudio el promedio de leucocitos fue de $17 \times 10^{3} / \mu \mathrm{L}$, encontrando que los pacientes con leucocitosis menor a $15 \times 10^{3} / \mu \mathrm{L}$ requirieron con mayor frecuencia nefrectomía $(p=$ $0.01)$. Posiblemente porque los pacientes tuvieron una mejor respuesta inmunológica e hicieron que el proceso infeccioso se limitara.

Los pacientes con una cuenta plaquetaria menor a $140 \times 10^{3} / \mu$ tienen mayor riesgo de desarrollar choque séptico $(p=0.027)$. En los pacientes con trombocitopenia menor a $50 \times 10^{3} / \mu \mathrm{L}, 90 \%$ requirió nefrectomía, manejo en terapia intensiva el $60 \%$ y desarrollaron choque séptico el $50 \%$ de ellos.

Los abscesos perirrenales pueden ser causados por diferentes tipos de microorganismos, siendo Gram negativos aerobios en $52 \%$ de los casos y predominando en estos Escherichia coli; el staphylococcus aureus se encuentra en $29 \%$ y los anaerobios en $17 \%$. Existen otros microorganismos menos frecuentes, Süleyman Kilic y cols. (10) reportaron un caso de

TABLA II. BACTERIAS ENCONTRADAS EN CULTIVOS DE MATERIAL PURULENTO ASPIRADO DURANTE EL DRENAJE QUIRÚRGICO.

\begin{tabular}{|l|c|}
\hline BACTERIA & $\begin{array}{c}\text { NÚMERO DE } \\
\text { PACIENTES (\%) }\end{array}$ \\
\hline ESCHERICHIA COLI & $10(43.47)$ \\
\hline KLEBSIELLA NEUMONIAE & $4(17.39)$ \\
\hline CANDIDA ALBICANS & $3(13.04)$ \\
\hline SIN DESARROLLO & $6(26.08)$ \\
\hline
\end{tabular}

absceso perirrenal causado por Salmonella enteritidis, en un paciente posterior a perforación del colon descendente. Existen reportes de abscesos perirrenales causados por Candida albicans (11). En $71 \%$ de los casos se desarrolla solo un microorganismo y en $29 \%$ dos o más $(12,13)$. En nuestro estudio se desarrolló Escherichia coli en 10 pacientes $(43.47 \%)$, Klebsiella neumoniae en 4 pacientes (17.31\%), Candida albicans en 3 pacientes (13.04\%) y 6 cultivos sin desarrollo (26.08\%).

El drenaje abierto es la modalidad que tiene mayor probabilidad de resolver el absceso perirrenal. En nuestra serie todos los pacientes se trataron con drenaje abierto, probablemente por lo avanzado de la enfermedad, ya que en todos los pacientes se encontró una cantidad de material purulento mayor a $50 \mathrm{ml}$ y en el $65 \%$ de los pacientes se drenó más de $200 \mathrm{ml}$.

Bickel y cols. (19) reportaron un caso de absceso perirrenal que fue tratado por laparoscopia. Mencionan en este reporte que a pesar de no ser el mejor abordaje, es una opción cuando el diagnóstico se realiza durante la exploración laparoscópica, pudiéndose tratar de forma satisfactoria.

Hasta una tercera parte de los pacientes pueden requerir nefrectomía parcial o total durante su estancia hospitalaria como parte del abordaje terapéutico inicial o de manera tardía $(1,20)$. En nuestro estudio el $78.3 \%$ de los pacientes requirió nefrectomía.

La mortalidad en nuestro estudio fue baja, de $8.69 \%$ probablemente debido a que nuestros pacientes consultan mas tardíamente y presentan una enfermedad más avanzada, teniendo que realizar un tratamiento más agresivo, además del manejo multidisciplinario que incluye el apoyo de terapia intensiva, consiguiendo una mortalidad más baja que la reportada en la literatura que va del $12-56 \%$ $(1,2,5,12)$. Los dos pacientes fallecidos tenían diagnóstico de diabetes mellitus con más de 10 años de evolución, uno de ellos con antecedente de litiasis renal, ambos con presentación inicial de fiebre mayor a $38.5^{\circ} \mathrm{C}$, con anemia, leucocitosis mayor a 16 $\times 10^{3} / \mu \mathrm{L}$, trombocitopenia menor a $130 \times 10^{3} / \mu \mathrm{L}$ y sodio sérico menor a $125 \mathrm{mEq} / \mathrm{L}$. Al realizar el análisis estadístico de los resultados de laboratorio asociados a mayor mortalidad únicamente el sodio bajo fue estadísticamente significativo $(p=0.038)$. A los dos pacientes se les realizó nefrectomía, manejándose en terapia intensiva, presentando neumonía nosocomial asociado a choque séptico, uno con 26 días de estancia en terapia intensiva y otro con 8 días. 
Del total 10 pacientes $(43.47 \%)$ fueron manejados en terapia intensiva, con una estancia promedio en terapia de 12 días y de estancia hospitalaria promedio de 24 días. La neumonía nosocomial se presentó en 8 pacientes (34.78\%), siete la adquirieron en la terapia intensiva, con una estancia hospitalaria promedio de 25 días.

El choque séptico se presentó en 8 pacientes $(34.78 \%)$ todos manejados en terapia intensiva, solamente las plaquetas menores a $140 \times 10^{3} / \mu \mathrm{L}$ se asociaron significativamente al desarrollo de este $(p=0.027)$.

La estancia hospitalaria promedio de estos pacientes es según Wheat y cols. (4) de 11 días, Meng y cols. (1) reportan 30 días en promedio, en nuestra serie fueron 20 días el promedio, con un mínimo de 5 y un máximo de 47 días.

\section{CONCLUSIONES}

En nuestra serie los principales factores predisponentes para la formación de abscesos retroperitoneales son: el género femenino, sobrepeso, diabetes mellitus y litiasis renal.

La fiebre al ingreso se presentó en la mayoría de los pacientes. La nefrectomía es necesaria en la mayoría de los pacientes con absceso perirrenal, siendo la hemoglobina mayor a $10.5 \mathrm{~g} / \mathrm{dL}$ y la cuenta leucocitaria menor a $15 \times 10^{3} / \mu \mathrm{L}$ factores asociados con mayor frecuencia a la pérdida de la unidad renal.

La cuenta plaquetaria menor a $140 \times 10^{3} /$ $\mu \mathrm{L}$ fue un factor estadísticamente significativo para el desarrollo de choque séptico. La estancia promedio en terapia intensiva fue de 12 días, desarrollando neumonía nosocomial el $70 \%$ de los pacientes.

La estancia hospitalaria promedio de los pacientes fue de 21 días y los factores asociados a mayor estancia hospitalaria fueron: hemoglobina menor a $11 \mathrm{~g} / \mathrm{dl}$, creatinina mayor a $2.5 \mathrm{mg} / \mathrm{dl}$, trombocitopenia menor a $50 \times 10^{3} / \mu \mathrm{L}$, glucemia mayor a $400 \mathrm{mg} / \mathrm{dl}$.

La mortalidad en nuestro estudio fue baja de $8.69 \%$ (2 pacientes), muy probablemente es el reflejo de la alta incidencia de nefrectomías y el apoyo expedito por el servicio de terapia intensiva. Al analizar la asociación de los estudios de laboratorio con la mortalidad únicamente el sodio menor a 125 $\mathrm{mEq} / \mathrm{L}$ fue estadísticamente significativo.

\section{BIBLIOGRAFÍA y LECTURAS RECOMENDADAS} (*lectura de interés $y^{* *}$ lectura fundamental)

**1. MENG, M.; MARIO, L.; MCANINCH, J.: "Current treatment and outcomes of perinephric abscesses". J. Urol., 168: 1337, 2002.

2. MERIMSKY, E.; FELDMAN, C.: "Perinephric abscess: Report of 19 cases". Int. Surg., 66: 79, 1981.

*3. ANDERSON, K.A.; MCANINCH, J.: "Renal abscesses: Clasification and review of 40 cases". Urology, 16: 333, 1980.

4. WHEAT, L.J.: "Infection and Diabetes mellitus". Diabetes Care, 3: 187, 1980.

**5. SHU, T.; GREEN, J.M.; ORIHUELA, E.: "Renal and perirenal abscesses in patients with otherwise anatomically normal urinary tracts". J. Urol., 172: 148,2004

*6. ANGEL, C.; SHU, T.; GREEN, J. y cols.: "Renal and perirenal abscesses in children proposed physiopathologic mechanisms and treatment algorithm". Pediatr. Surg. Int., 19: 35, 2003.

7. KAWANO, A. y cols.: "Serious renal infection: Report of three cases". Hinyokika Kiyo, 49: 207, 2003.

*8. SIEGEL, J.F.; SMITH, A.; MOLDWIN, R.: "Minimally invasive treatment of renal abscess". J. Urol., 155: 52, 1996.

9. BONVIN, B.; VON BUREN, U.: "A urologic-radiological view of perinephric abscess". Rofo, 124: 358, 1976.

10. SÜLEYMAN, K.; MEHMET, R.T.; HÜSEYIN, E. y cols.: "Left perinephric abscess caused by salmonella enteritidis due to colon perforation". J. Urol., 170: 1945,2003

11. TSAI, S.H.; PENG, Y.J.; WANG, N.C.H.: "Pyomyositis with hepatic and perinephric abscesses caused by candida albicans in a diabetic nephropathy patient". American Journal of the Medical Sciences, 331: 292, 2006

**12. CAPITAN MANJON, C.; TEJIDO SÁNCHEZ, A.; PIEDRALARA, J.D. y cols.: "Retroperitoneal abscesses análisis of a series of 66 cases". Scan. J. Urol. Nephrol., 37: 139, 2003.

*13. EDELSTEIN. H.; McCABE, R.E.: "Perinephric abscess. Modern diagnosis and treatment in 47 cases". Medicine, 67: 118, 1988 .

14. TUNUGUNTLA, A.; RAZA, R.; HUDGINS, L.: "Diagnostic and therapeutic difficulties in retroperitoneal abscess". Southern Medical Journal, 97: 1107, 2004.

**15. SCHAEFFER, A.J.: "Infections of the urinary tract". Walsh P.C., Retik A.B., Vaughan Jr E.D., Wein A.J., editors. Campbell's urology. 8th ed. Philadelphia: W.B. Saunders, p. 558-563, 2002.

16. SCHIFF, M.JR.; GLICKMAN, M.; WEISS, R.M. y cols.: "Antibiotic treatment of renal carbuncle". Ann. Intern. Med., 87: 305, 1977.

17. SAMDAL, F.; VADA, K.; LOE, B. y cols.: "Perinephric abscess". Tidsskr Nor Laegeforen, 110: 1952, 1990.

18. RIVES, R.K.; HARTY, J.I.; AMIN, M.: "Renal abscess: Emerging concepts of diagnosis and treatment". J. Urol., 124: 446, 1980.

*19. BICKEL, A.; WAXMAN, I.; EITAN, A.: "Laparascopic treatment of a perinephric abscess". Surgical endoscopy, 9: 437, 1995.

20. DEMBRY, L.M.; ANDRIOLE, A.T.: "Renal and perirenal abscesses". Infect. Dis. Clin. North Am., 11: $663,1997$. 